\title{
Effects of flu vaccine, solely or accompanied by pneumovax-23 vaccine on clinical consequences of the respiratory diseases among Iranian pilgrims in
} Hajj

\author{
Soheyla Dabiran ${ }^{1}$, Seyed Mansour Razavi ${ }^{2}$, Ahmad Sabouri Kashani ${ }^{3}$, Mojgan Karbakhsh ${ }^{1}$, \\ Haleh Naser-Hodjati ${ }^{4}$, Far Peyafarin ${ }^{5}$ \\ ${ }^{1}$ Department of Community Medicine, Tehran University of Medical Sciences, Tehran, Iran; \\ dabirans@Sina.TUMS.ac.ir, kabakh@Sina.TUMS.ac.ir \\ ${ }^{2}$ Research Center for Rational Use of Drugs, Tehran University of Medical Sciences, Tehran, Iran \\ *Corresponding Author: Razavy@Sina.TUMS.ac.ir \\ ${ }^{3}$ Educational Development Center, Tehran University of Medical Sciences, Tehran, Iran; Sabourika@Yahoo.com \\ ${ }^{4}$ Clinical Skills Training Center, Tehran University of Medical Sciences, Tehran, Iran; Haleh337@Yahoo.com \\ ${ }^{5}$ Private Section, Tehran, Iran
}

Received 26 November 2013; revised 25 December 2013; accepted 15 January 2014

Copyright (C) 2014 Soheyla Dabiran et al. This is an open access article distributed under the Creative Commons Attribution License, which permits unrestricted use, distribution, and reproduction in any medium, provided the original work is properly cited. In accordance of the Creative Commons Attribution License all Copyrights (c) 2014 are reserved for SCIRP and the owner of the intellectual property Soheyla Dabiran et al. All Copyright @ 2014 are guarded by law and by SCIRP as a guardian.

\section{ABSTRACT}

Background: Respiratory disorders are the most common diseases among Iranian pilgrims in Hajj. Some studies recommended flu and pneumococcal vaccines separately or in combination to reduce respiratory diseases occurrence and its morbidity. These effects are not clear in afflicted Hajj pilgrims. Objective: This is a cohort study that investigates the effects of above-mentioned vaccines each or their combination on respiratory syndromes and their consequences among Iranian pilgrims in Mecca. Method: The participants were 295 Iranian pilgrims from the beginning to the end of the journey. They were systematically observed for getting afflicted with a respiratory syndrome or not. In this study, we have assessed the effects of flu and pneumococcal vaccines on respiratory disease occurrence and on 8 clinical consequences including as: the duration of fever, duration of cough, duration of bed rest, presence of post nasal discharge and dirty sputum, the total disease length, and the number of referrals to the hospital, physician's visits and complications. The data for this study were gathered through a questionnaire with 14 basic questions and 60 choices. This was processed using the SPSS software of
11.5 version, T- and Chi-square tests. Results: The results showed no significant correlations between the above-mentioned factors and vaccination with one or both flu and pneumovax-23 except for the duration of coughs which was significantly reduced in all cases $(p<0.01)$ and also decreased complications of the disease $(p<$ 0.001). Conclusion: The study suggests the highrisk groups, should be vaccinated with combination of flu and pneumovax-23 vaccines in Hajj.

\section{KEYWORDS}

Respiratory Disease; Flu Vaccine;

Pneumovax-23; Clinical Outcomes; Hajj

\section{INTRODUCTION}

Every year more than 2 million Muslims from 140 different countries go on a pilgrimage to Saudi Arabia [1]. Some respiratory tract involvements originating from different sources have been most common among the pilgrims. In a study during Hajj 2005, 70\% of Iranian pilgrims were afflicted with some types of respiratory disorders, in $50.8 \%$ of whom common cold syndrome was found responsible, $19.6 \%$ were due to influenza like illness, allergic syndromes comprised $31.3 \%$, pyogenic pharyngitis $7.9 \%$, acute sinusitis, recurrence of previous 
cases and sinobronchitis 9.9\%, COPD and asthma 1.48\% and finally pneumonia $0.34 \%$ [2].

In another study, the high-risk Iranian pilgrims were estimated to form $25.6 \%$ of the total number of this population. These were usually afflicted with a number of such diseases as hypertension, diabetes, cardiovascular diseases, asthma, COPD, and sinusitis; they belonged to the above 65 age group and suffered from some underlying disabilities [3].

Since the incidence of respiratory involvements among pilgrims is high and on the other hand, high risk individuals are more at risk for infections than other people, for reducing respiratory diseases occurrence we should consider some effective preventive programs in our agenda during Hajj.

Some studies recommended flu and pneumococcal vaccines separately or in combination to reduce respiratory diseases occurrence; reducing mortality and bedrest duration in hospitals [4,5]; reducing morbidity, disease recurrence, or complications and disabilities [6]; reducing bed rest, and enhancing convalescence [7]; also reducing complications in persons who suffer from diabetes mellitus, cardiac diseases, COPD, and renal, or hepatic diseases $[8,9]$.

No specific studies about reducing the adverse consequences of respiratory infections by these two vaccines were found in the Hajj.

In the present study, the effects of flu vaccine solely or accompanied by pneumococcal vaccine (pneumovax-23) on the clinical manifestations progress (clinical consequences) among involved pilgrims were assessed.

\section{MATERIALS AND METHODS}

This cohort study was conducted on 325 volunteered Hajj pilgrims in the year 2005. In this study, two weeks before the journey, we administered one dose $(0.5 \mathrm{ml})$ of Flu-vaccine solely or with $0.5 \mathrm{ml}$ Pneumovax-23 simultaneously to the volunteers on the basis of physician's decision.

Each dose of Flu-vaccine contained, three different types of influenza viruses, prepared according to antigenic changes in the year 2004 and also each 0.5 milliliter of Pneumovax-23, contained $0.025 \mathrm{mg}$ of purified capsular polysaccharide from several Streptococcus pneumonia serotypes as: 1, 2, 3, 4, 5, 6B, 7F, 8, 9N, 10A, 11A, 12F, 14, 15B, 17F, 18C, 19F, 20, 22F, 23F and was applied intramuscularly in Deltoid muscle. Volunteers were monitored for respiratory disorders from a couple of weeks before their pilgrimage, through the pilgrimage and up to the end of their trip when they left Saudi Arabia (at least 45 days). On their arrival at Saudi Arabia, 30 of the candidates were excluded from the study as they suffered from some respiratory disorders. The remaining 295 participant, who lacked any signs or symptoms of respirato- ry syndromes, were closely monitored by their Caravan's physician throughout their one-month pilgrimage.

Each participant was primarily given a thorough check up followed by close monitoring of involvement to a respiratory syndrome or not and in case of disease occurrence, monitoring of some clinical Consequences and ultimately, evaluation of relationships between vaccines, disease occurrence and clinical consequences.

Clinical consequences that were assessed were 8 outcomes including: 1) Periods of fever; 2) Duration of coughs; 3) Presence of dirty post nasal discharge (PND); 4) Duration of bed rest at the Caravan; 5) The length of time taken for the sputum/nasopharynx secretions to change color from yellowish-greenish to a clear liquid; 6) Number of visits; 7) The total period of the disease; and 8) The existence of any such complications as (Exudative sore throat, Purulent conjunctivitis, Sinusitis or Recurring sinusitis, Delayed coughs with purulent sputum, Pneumonia and the infection of the middle ear), based on the physicians report.

High risk individuals in this study were the pilgrims who were suffering from one or more chronic diseases such as: hypertension, diabetes mellitus, renal, hepatic, pulmonary or cardiac diseases or were older than 65 years of age.

The data were gathered through a questionnaire containing 14 main questions with 60 options. The questionnaire had already been validated by a group of experts and its reliability was confirmed by a pilot study in the previous years.

All Caravans' physicians had training workshops upon their arrival at Saudi Arabia to correctly fill in the questionnaires or supervise data collection. After going through different stages of data grouping, data checking, data cleaning, and data completing, the data were analyzed by SPSS v. 11.5 making use of Student $t$, and Chi square tests.

\section{RESULTS}

During the Hajj of 2006, 295 volunteered Iranian pilgrims were monitored for any respiratory syndromes during their one-month pilgrimage to Mecca. None of the participants suffered from any kind of respiratory disorders on their arrivals. There were 127 females (43.1\%) and 168 males (56.9\%). The average age of the participants was 52.1 years $(S D=12.6)$ with a range of $20-85$ years of age.

There were 99 high risk cases included (33.5\%), who were suffering from one or more chronic diseases such as: hypertension, diabetes mellitus, renal-hepatic or cardiac diseases or were older than 65 years of age, and 196 of them (66.5\%) were quite healthy. Out of these, 78 people (26.4\%) had only one of the two vaccines, either the flu vaccine or the pneumovax-23, injected 2 weeks before their departure to Mecca, while 188 of them (63.7\%) had the two vaccines combined, and 19 participants (6.4\%) 
had neither. The data regarding 10 of the participants (3.5\%) were incomplete.

During the pilgrimage 54 people (18.4\%) did not show any signs or symptoms of respiratory disorders. Physicians' reports did not mention anything on 9 (3\%) of the participants (Missing cases). But we have detailed information on 232 cases (78.6\%), who did suffer from some sort of respiratory disorders. Out of these people, 104 cases (35.2\%) started before going to Arafat, Mash, ar \& mina (Holy hillsides around the Mecca) and 156 cases (52.8\%) afterwards. We have not enough information about 35 (12\%) people.

The frequency of the observed symptoms for those afflicted with some sort of respiratory diseases are shown in Table 1.

The mean lengths of time for each clinical consequences (clinical outcomes) has been shown in Table 2. Frequencies of applied vaccines according to existence of respiratory complications (Pneumonia, Conjunctivitis, Otitis, Sinusitis and Pharyngitis) according to physician's report is shown in Table 3 and also the correlation between applying of Flu vaccine solely or accompanied by Pneumovax-23 and duration of cough among understudied population were shown in Table 4 .

\section{DISCUSSION}

In this study, 295 pilgrims (43.1\% females and 56.9\% males) were monitored during one month in Hajj 2006 for the occurrence of respiratory syndromes, clinical outcomes and determination of correlation between vaccine applying and these factors.

Table 1. Frequency of symptoms of “respiratory diseases” observed among the understudied Iranian pilgrims in Hajj 2006.

\begin{tabular}{|c|c|c|c|c|c|}
\hline Symptoms & Percentage & Symptoms & Percentage & Symptoms & Percentage \\
\hline Runny nose & 51.7 & Sore Throat & 34.2 & Back pain & 13.8 \\
\hline Dry coughs & 51.7 & Painful swallowing & 23.8 & Clear PND & 13.8 \\
\hline Hoarseness & 47.7 & Throat itch & 28.6 & Arthralgia & 11.7 \\
\hline Yellowish-greenish sputum & 42.8 & Fever & 26.8 & Clear sputum & 9.8 \\
\hline Nose congestion & 38.8 & Muscular pain (myalgia) & 24.6 & Shivering & 4.9 \\
\hline Purulent PND & 35.5 & Feeling chills & 17.8 & Eye infection & 4.6 \\
\hline
\end{tabular}

$\mathrm{N}=295$; a patient may be suffering from one or more symptoms or signs.

Table 2. The mean lengths of time for each clinical outcome among Iranian pilgrims during Hajj 2006.

\begin{tabular}{|c|c|c|c|c|c|}
\hline Clinical outcomes (consequences) & No of investigated cases. & $\begin{array}{l}\text { Average mean of length } \\
\text { of coughs (in days) }\end{array}$ & SD & Minimum & Maximum \\
\hline Duration of fever & 100 & 3.5 & 4.2 & 1 & 25 \\
\hline Duration of cough & 192 & 8.1 & 6.5 & 1 & $>30$ \\
\hline Duration of PND & 149 & 8.3 & 6.25 & 1 & 30 \\
\hline Duration of bed rest at the Caravan & 30 & 2.8 & 2.32 & 1 & 12 \\
\hline Duration of the color of sputum or PND change to clear & 97 & 6.8 & 5.75 & 1 & 25 \\
\hline Total period of the disease & 205 & 8.3 & 7.3 & 1 & $>30$ \\
\hline Number of visits by physicians & 232 & 2.9 & 1.75 & 1 & 10 \\
\hline
\end{tabular}

Table 3. The frequencies of applied vaccines according to existence of respiratory complications among the under studied pilgrims.

\begin{tabular}{|c|c|c|c|c|c|c|}
\hline \multirow{2}{*}{$\begin{array}{c}\text { Complication* } \\
\text { type of vaccination }\end{array}$} & \multicolumn{2}{|c|}{ Yes } & \multicolumn{2}{|c|}{ No } & \multicolumn{2}{|c|}{ Total } \\
\hline & $\mathbf{N}$ & $\%$ & $\mathbf{N}$ & $\%$ & $\mathbf{N}$ & $\%$ \\
\hline Flu vaccine & 20 & 24.1 & 63 & 75.9 & 83 & 100 \\
\hline Flu + pneumovax & 32 & 17 & 156 & 83 & 188 & 100 \\
\hline None & 10 & 52.6 & 9 & 47.4 & 19 & 100 \\
\hline Missing & \multicolumn{2}{|c|}{2} & \multicolumn{2}{|c|}{295} & \multicolumn{2}{|c|}{100} \\
\hline
\end{tabular}

*Exudative sore throat, purulent conjunctivitis, acute sinusitis or recurring sinusitis, delayed coughs with purulent sputum, pneumonia and the infection of the middle ear. 
Table 4. Correlation between applying of flu vaccine solely or accompanied by pneumovax-23 and duration of cough among understudied population.

\begin{tabular}{cccccc}
\hline Type of vaccination & No & Average mean of length of coughs (in days) & SD & Minimum & Maximum \\
\hline Flu & 58 & 8.4 & 6.2 & 1 & 25 \\
Flu + pneumovax & 119 & 7.5 & 6.5 & 1 & 38 \\
None & 11 & 13.7 & 6.4 & 3 & 22 \\
Total & 188 & 8.1 & 6.6 & 1 & 38 \\
\hline
\end{tabular}

$\mathrm{p}=0.001$.

The mean age of the group was 52.1 years $33.5 \%$ of whom belonged to the high risk group.

In one similar study that conducted in the year of 2003, there were 30,037 Iranian pilgrims (54.7\% males and $45.3 \%$ females) with a mean age group of 53.5 years [3]. Our understudied population were a little younger than above mentioned one.

In previous study, with a similar definition for the high risk groups, $25.6 \%$ of the pilgrims were comprised and the relationship between susceptibility among high risk group, and respiratory involvement were also significant in this study [3]. In our study, the rate of the high risk group were higher than above mentioned study (33.5\% versus $25.6 \%$ ), therefore it seems our understudied population were more at risk. This higher susceptibility may be attributed to the fact that those who were more susceptible were more willing to volunteer to be closely monitored for their health throughout the journey.

In the present study, 232 (78.6\%) pilgrims were afflicted with some sort of respiratory disorders while this figure was $70 \%$ in one quiet similar study [12] and 35\% in another resembling study with similar setting [10], which shows a much higher level of respiratory involvement in our understudied population. Perhaps, this finding is due to high level of high risk group in our study.

In one study performed in Finland in 2001, 76 young healthy men were assessed for common cold clinical manifestations progress. In that study, the duration of the cough had an average of 8.4 days with an SD of 6.5. Interestingly, the results were very similar to those of our study.

However, the average period of producing sputum was 5.9 days, with an SD of 6.4 which took 21 days to recover without prescribing antibiotics [11], two indicators far from those of ours.

The effectiveness of the flu vaccine in reducing respiratory syndromes among pilgrims is controversial. Some studies found it effective [12] while some others useless [2]. However, pneumovax has proved to be effective [5, $8,13-15]$. In the present study, the flu vaccine and pneumovax each was found effective and the latter was apparently even more effective in reducing respiratory disorders. Yet, the effect was statistically insignificant, which might be assigned to the small sample size. Influenza vaccine, solely, or combined with pneumovax, significantly reduced the average period of coughs. Nevertheless, the reduction in other symptoms was not significant.

Among the limitations of the study, we may mention lacking sufficient time to get the consent of a larger number of candidate pilgrims, lack of easy access to the candidates, occasional cases lasting more than one month, and losing some suspected data. There was no any ethical problem in this study.

\section{CONCLUSIONS}

This study concluded that, administration of flu-vaccine solely or accompanied by pnemovax-23, decrease the mean of duration of the fever, cough, post nasal discharge, dirty sputum, bed rest, number of visits by physicians and total period of the disease.

The effect of these vaccines on reducing of cough duration was significant $(p=0.001)$. Therefore, the researchers suggest that the Hajj health managers, should consider the combined vaccination of both pneumovax-23 and flu vaccines in preventive protocol of the Iranian high-risk groups in Hajj.

\section{ACKNOWLEDGEMENTS}

We should, hereby thank all Caravans' physicians who sincerely cooperated to accomplish the objectives of the project. Thanks are due to all the health workers who helped collecting the recorded data. Special thanks are extended to Dr Hollysaz, Dr. Rajayee, Dr. Taamoli, Dr. Sadeghipoor, Dr. Shojayeezadeh, Ms. Lashgari, Ms. Mohammadi, and Ms. Chari.

\section{REFERENCES}

[1] Centers for Disease Control and Prevention (CDC) (2001) Risk for meningococcal disease associated with the Hajj 2001. Morbidity and Mortality Weekly Report, 50, 97-98.

[2] Razavi, M., Sadeghi-Hassanabadi, M. and Salamati, P. (2005) The comparison of influenza vaccine efficacy on respiratory disease among Iranian pilgrims. Acta Medica Iranica, 43, 1-3.

[3] Meysamie, A., Ardakani, H.Z., Razavi, S.M. and Doroodi, T. (2006) Comparison of mortality and morbidity rates among Iranian pilgrims in Hajj 2004 and 2005. Saudi Medical Journal, 27, 447-451.

[4] Rubins, J.B. and Janoff, E.N. (2001) Pneumococcal dis- 
ease in the elderly: What is preventing vaccine efficacy? Drugs Aging, 18, 305-311. http://dx.doi.org/10.2165/00002512-200118050-00001

[5] Vila-Corcoles, A., Ochoa-Gondar, O., Hospital, I., Ansa, X., Vilanova, A., Rodriquez, T. and Llor, C., Evan Study Group (2006) Protective effects of the 23-valent pneumococcal polysaccharide vaccine in the elderly population. Clinical Infectious Diseases, 43, 860-886. http://dx.doi.org/10.1086/507340

[6] Granger, R., Walters, J., Poole, P.J., Lesserson, T.J., Manqtani, P., Cates, C.J. and Wood-Baker, R. (2006) Injectable vaccines for preventing pneumococcal infection in patients with chronic obstructive pulmonary disease. Cochrane Database of Systematic Reviews, 11, Article ID: CD00139.

[7] Mykietiuk, A., Garratala, J., Domingez, A., Manzur, A., Fernandez-Sabe, N., Dorca, J., Tubau, F., Mensesa, F. and Gudiol, F. (2006) Effect of prior pneumococcal vaccination on clinical outcome of Hospitalized adults with community-acquired pneumococcal pneumonia. European Journal of Clinical Microbiology \& Infectious Diseases, 25, 457-462. http://dx.doi.org/10.1007/s10096-006-0161-8

[8] Bulter, J.C., Breiman, R.E., Campbell, J.F., Lipman, H.B., Broome, C.V. and Facklam, R.R. (1993) Pneumococcal polysaccharide vaccine efficacy. An evaluation of current recommendations. JAMA, 270, 1826-1831. http://dx.doi.org/10.1001/jama.1993.03510150060030

[9] Centers for Disease Control and Prevention (CDC) (2006) Influenza and pneumococcal vaccination coverage among persons aged > 65 years in United States, 2004-2005. Morbidity and Mortality Weekly Report, 55, 1065-1068.

[10] Razavi, S.M., Ziaee, H. and Sedaghat, M. (2004) Morbidity and mortality among Iranian pilgrims and factors involved, in Hajj 2003. Journal of the Faculty of Medicine, 62, 1 .

[11] Puhakka, T., Lavonius, M., Varpula, M., Svedstrom, E., Terho, E. and Ruuskanen, O. (2001) Pulmonary imaging and function in the common cold. Scandinavian Journal of Infectious Diseases, 33, 211-214. http://dx.doi.org/10.1080/00365540151060888

[12] Razavi, S.M., Dabiran S. and Ardekani, H.Z. (2004) The incidence of influenza like Illness and determination of the efficacy of fluvaccine in Iranian pilgrims during Hajj pilgrimage. Acta Medica Iranica, 42, 397-401.

[13] Spike, J.S., Fedson, D.S. and Facklam, R.R. (1990) Pneumococcal vaccination; controversies and opportunities. Infectious Disease Clinics of North America, 4, 11-27.

[14] Ansaldi, F., Turello, V., Lai, P., Bastone, G., Deluca, S., Rossell, R., Durando, P., Sticch, L., Gasparini, R., Delfino, E. and Icardi, G. (2005) Effectiveness of a 23-valent polysaccharide vaccine in preventing pneumonia and non-invasive pneumococcal infection in elderly people. Journal of International Medical Research, 33, 490-500. http://dx.doi.org/10.1177/147323000503300503

[15] Melton, K.A. (2001) Pneumococcal vaccine. Primary Care Update for $O B / G Y N S$, 8, 44-47. http://dx.doi.org/10.1016/S1068-607X(00)00070-6 